\title{
Considering Late-Time Acceleration in Some Cosmological Models
}

\author{
S. Davood Sadatian \\ Department of Physics, Faculty of Basic Sciences, University of Neyshabur, P.O. Box 91136-899, Neyshabur, Iran \\ Correspondence should be addressed to S. Davood Sadatian; sd-sadatian@um.ac.ir
}

Received 14 February 2013; Revised 12 March 2013; Accepted 14 March 2013

Academic Editor: Kingman Cheung

Copyright (c) 2013 S. Davood Sadatian. This is an open access article distributed under the Creative Commons Attribution License, which permits unrestricted use, distribution, and reproduction in any medium, provided the original work is properly cited.

\begin{abstract}
We study two cosmological models: a nonminimally coupled scalar field on brane world model and a minimally coupled scalar field on Lorentz invariance violation model. We compare some cosmological results in these scenarios. Also, we consider some types of Rip singularity solution in both models.
\end{abstract}

\section{Introduction}

In theories of extra spatial dimensions, ordinary matter is captured on the brane but gravitation extends through the entire space time [1-3]. In these scenarios, the cosmological evolution on the brane is taken by an effective Friedmann equation that combines the effects of the bulk in a nontrivial kind [4-6]. In other view point, the important result in brane models is an alternative scenario for late-time expansion of the universe. This result predicts deviations from the 4-dimensional gravity at limited distances. In other hand, the model considered by Dvali, Gabadadze, and Porrati (DGP) is different from previous models since it also predicts deviations from the standard 4-dimensional gravity in large distances [4,7]. Generally one can study the effect of a caused gravity term as a quantum discipline in any brane world model. The existence of a higher dimensional embedding space lets for the bulk or brane matter that can evidently affect the cosmological evolution on the brane [8]. A special form of bulk or brane context is a scalar field. Scalar fields take an important key both in models of the early universe and latetime acceleration. These scalar fields give a dynamical model for matter fields in a brane world scenarios [9-12].

In other hand, Lorentz invariance violation models (LIV) have been considered in the scalar-vector-tensor theories [13]. It has described that Lorentz violating vector fields influence the dynamics equations in the inflationary models. An interesting result of this model is that the exact Lorentz violating inflationary solutions are dependent on the absence of the inflation potential. In this case, the inflation is exactly collaborated with the Lorentz violation [14]. Therefore, we study this symmetry breaking on the dynamics of equation of state for some cosmological aspects.

However, some evidence from supernova data $[15,16]$, (CMB) results [17-19] and (WMAP) data [20-24] points out an accelerating phase of cosmological expansion, and this characteristic shows that the picture of universe by pressureless fluid is not enough; the universe should contain some type of additional negative pressure known as dark energy (for brief introduction in this field see [14]). Also, the merged analysis of the WMAP data with the supernova Legacy survey (SNLS) [22] compels the equation of state $w_{d e}$, in accordance with $74 \%$ donation of dark energy in the currently accelerating universe. Moreover, observations show some sort of a dark energy equation of state, $w_{d e}<-1$ [23]. Hence, a practical cosmological model should accept a dynamical equation of state that may have crossed the value $w_{d e}=-1$, in late time of cosmological evolution.

In the following, we consider cosmological results of a nonminimally coupled scalar field on the brane and a minimally coupled scaler field in LIV model. Also we determine late-time behavior of our equations and obtain some restriction on the parameters of models to have an accelerating universe.

We understand that dark energy is an important problem in modern cosmology, especially, if the equation of state parameter $\omega$ is less than -1 . In this paper, we study basically solving Friedman equations and obtain the evolution of the 
effective equation of state parameter $\omega$. The phantom crossing conditions that we obtained for these set-ups, are interesting at some degree themselves. Furthermore, we know that just considering background quantity is not enough in such kind of unconventional cosmological models. The current constraint on $\omega$ is obtained by combining the result of the observation of CMB, which means that the information of the evolution of perturbations should be also included. Also we understand in the brane world model that the perturbations of brane are coupled with these of bulk, which gives nontrivial effect. Therefore, in first stage we just study the background dynamics, and the evolution of perturbations will be studied in the future.

In other view, there is a no-go theorem which explicitly points out that a conventional dark energy model involving single degree of freedom in the frame of standard Einstein gravity is forbidden to realize such a scenario. This no-go theorem was proven in the appendix of [25]. In this regards, we point out the original work in [26] and several papers addressing this scenario [27-29]. This theorem is exactly the reason why we study a number of nonconventional dark energy models in realizing the Quintom scenario [24], such as the nonminimally coupled on brane world model and the Lorentz-violating model considered in the present paper. However, a nonminimally coupling scalar field identified on the brane model and scalar field coupling minimally to gravity in LIV model have some similar cosmological results; this is an interesting theoretical motivation of these models. Also we study other solutions admitted as Rip singularity that occur in the condition $\omega<-1$ increases rapidly. However, it possible different types of singularity, dependingenergy density and scale factor how increases with time $[30,31]$.

\section{Nonminimally Coupled Scalar Field on the Brane}

Here we study a brane world model where a scalar field is coupling nonminimally to the Ricci scalar of the brane. In the following we only consider a scalar field in the matter Lagrangian without taking into account baryons, cold dark matter, and radiation. This kind of analysis suffers from a potential that may cause that the model is not able to explain the evolution of a realistic universe at background level when confronting with observations. However, this problem can be solved by suitable fine-tuning parameters of model.

The action in the absence of ordinary matter can be given as $[12,32]$

$$
S=\int d^{4} x \sqrt{-g}\left[\frac{1}{k_{4}^{2}} \alpha(\phi) R[g]-\frac{1}{2} g^{\mu \nu} \nabla_{\mu} \phi \nabla_{\nu} \phi-V(\phi)\right],
$$

where we have made a general nonminimal coupling $\alpha(\phi)$. For simplicity, in the following, we set $k_{4}^{2} \equiv 8 \pi G_{N}=1$. We can obtain Einstein equations with variation of the action with respect to brane metric

$$
R_{\mu \nu}-\frac{1}{2} g_{\mu \nu} R=\alpha^{-1} \mathscr{T}_{\mu \nu}
$$

where $\mathscr{T}_{\mu \nu}$, energy-momentum tensor of the scalar field, nonminimally coupled to gravity is taken by

$$
\begin{aligned}
\mathscr{T}_{\mu \nu}= & \nabla_{\mu} \phi \nabla_{\nu} \phi-\frac{1}{2} g_{\mu \nu}(\nabla \phi)^{2}-g_{\mu \nu} V(\phi) \\
& +g_{\mu \nu} \square \alpha(\phi)-\nabla_{\mu} \nabla_{\nu} \alpha(\phi),
\end{aligned}
$$

where $\square$ shows 4-dimensional d'Alembertian. We study the FRW universe with line element as the following:

$$
d s^{2}=-d t^{2}+a^{2}(t) d \Sigma_{k}^{2},
$$

where $d \Sigma_{k}^{2}$ is the line element for a constant curvature $k=$ $+1,0,-1$. The Ricci scalar is obtained with the equation of motion for scalar field as

$$
\nabla^{\mu} \nabla_{\mu} \phi=V^{\prime}-\alpha^{\prime} R[g],
$$

where a prime denotes the derivative of each parameter with respect to $\phi$, that may be written by

$$
\ddot{\phi}+3 \frac{\dot{a}}{a} \dot{\phi}+\frac{d V}{d \phi}=\alpha^{\prime} R[g],
$$

where a dot means the derivative of each parameter with respect to $t$. The intrinsic Ricci scalar for a FRW brane is given as

$$
R[g]=6\left(\dot{H}+2 H^{2}+\frac{k}{a^{2}}\right)
$$

and Friedmann's equations are determined by

$$
\begin{gathered}
\frac{\dot{a}^{2}}{a^{2}}=-\frac{k}{a^{2}}+\frac{\rho}{3}, \\
\frac{\ddot{a}}{a}=-\frac{1}{6}(\rho+3 p) .
\end{gathered}
$$

We take a scalar field, $\phi$, only dependent on time. So, with (3), we obtain

$$
\begin{gathered}
\rho=\alpha^{-1}\left(\frac{1}{2} \dot{\phi}^{2}+V(\phi)-6 \alpha^{\prime} H \dot{\phi}\right) \\
p=\alpha^{-1}\left(\frac{1}{2} \dot{\phi}^{2}-V(\phi)+2\left(\alpha^{\prime} \ddot{\phi}+2 H \alpha^{\prime} \dot{\phi}+\alpha^{\prime \prime} \dot{\phi}^{2}\right)\right),
\end{gathered}
$$

where $H=\dot{a} / a$ is Hubble parameter. Now equation of state has the following form:

$$
w \equiv \frac{p}{\rho}=\frac{\dot{\phi}^{2}-2 V(\phi)+4\left(\alpha^{\prime} \ddot{\phi}+2 H \alpha^{\prime} \dot{\phi}+\alpha^{\prime \prime} \dot{\phi}^{2}\right)}{\dot{\phi}^{2}+2 V(\phi)-12 \alpha^{\prime} H \dot{\phi}} .
$$

When $\dot{\phi}=0$, we have $p=-\rho$. In this illustration $\rho$ depended on $a$ and $V(\phi)$, and it has the duty of a cosmological constant. In the minimal case in which $\dot{\phi}^{2}<V(\phi)$, by (9), we take $p<-\rho / 3$ that shows a late-time accelerating universe. In nonminimal case, the position depends on the choice of nonminimal coupling parameter. In the following we show how for a suitable range of coupling parameter, a late-time accelerating expansion can be described. We first consider a moving domain wall of brane world to discuss quintessence behavior, and then we study a special nonminimal coupling for late-time acceleration. 
2.1. Late-Time Acceleration in a Brane World Model. We study a bulk configured by two 5-dimensional anti de SitterSchwarzschild $\left(\mathrm{AdSS}_{5}\right)$ black hole spaces combined on a moving domain wall. To insert this moving domain wall into 5-dimensional bulk, it is required to indicate normal and tangent to the domain wall by determination of normal instructing to the brane. We take that domain wall is identified at coordinate $r=a(\tau)$ where $a(\tau)$ is considered by Israel junction conditions [33]. Here we study the following line element [32]:

$$
\begin{aligned}
d s_{5 \pm}^{2}= & -\left(k-\frac{\eta_{ \pm}}{r^{2}}+\frac{r^{2}}{\ell^{2}}\right) d t^{2} \\
& +\frac{1}{k-\eta_{ \pm} / r^{2}+r^{2} / \ell^{2}} d r^{2}+r^{2} \gamma_{i j} d x^{i} d x^{j}
\end{aligned}
$$

where \pm is for left $(-)$ and right $(+)$ side of the moving domain wall, also $\ell$ is curvature radius of $\mathrm{AdS}_{5}$ manifold, and $\gamma_{i j}$ is the horizon metric. $\eta_{ \pm} \neq 0$ creates the electric part of the Weyl tensor on two sides [32].

We assume that usual mater on the brane has a perfect fluid form, $T_{\mu \nu}=(\rho+p) u_{\mu} u_{\nu}+p h_{\mu \nu}$ where $\rho=\rho_{m}+\sigma$ and $p=p_{m}-\sigma$. Energy density of the confined matter on the brane and analogous pressure is shown with $\rho_{m}$ and $p_{m}$, while $\sigma$ is the brane tension. with Israel junction conditions [33] and Gauss-Codazzi equations, we obtain generalized Friedmann equations [32] as the following:

$$
\begin{gathered}
\frac{\dot{a}^{2}}{a^{2}}+\frac{k}{a^{2}}=\frac{\rho_{m}}{3}+\frac{\eta}{a^{4}}+\frac{\ell^{2}}{36} \rho_{m}^{2}, \\
\frac{\ddot{a}}{a}=-\frac{\rho_{m}}{6}(1+3 w)-\frac{\eta}{a^{4}}-\frac{\ell^{2}}{36} \rho_{m}^{2}(2+3 w),
\end{gathered}
$$

where we require a $Z_{2}$-symmetry by $\eta_{+}=\eta_{-} \equiv \eta$, and we have supposed $p_{m}=w \rho_{m}$. Following we take case: $\eta=0$. For $\eta=0$, each submanifold of bulk space time is accurate $\mathrm{AdS}_{5}$ space time. Now we study a confined nonminimally coupled scalar field on the brane and consider cosmological aspects. We use energy density and pressure of scalar field (10), and by (14), hence for cosmic expansion we have

$$
\begin{aligned}
\frac{\ddot{a}}{a}= & -\frac{1}{6 \alpha}\left(\frac{1}{2} \dot{\phi}^{2}+V(\phi)-6 \alpha^{\prime} H \dot{\phi}\right) \\
& \times\left(1+3 \frac{\dot{\phi}^{2}-2 V(\phi)+4\left(\alpha^{\prime} \ddot{\phi}+2 H \alpha^{\prime} \dot{\phi}+\alpha^{\prime \prime} \dot{\phi}^{2}\right)}{\dot{\phi}^{2}+2 V(\phi)-12 \alpha^{\prime} H \dot{\phi}}\right) \\
& -\frac{\ell^{2}}{36 \alpha^{2}}\left(\frac{1}{2} \dot{\phi}^{2}+V(\phi)-6 \alpha^{\prime} H \dot{\phi}\right)^{2} \\
& \times\left(2+3 \frac{\dot{\phi}^{2}-2 V(\phi)+4\left(\alpha^{\prime} \ddot{\phi}+2 H \alpha^{\prime} \dot{\phi}+\alpha^{\prime \prime} \dot{\phi}^{2}\right)}{\dot{\phi}^{2}+2 V(\phi)-12 \alpha^{\prime} H \dot{\phi}}\right),
\end{aligned}
$$

where $H=\dot{a} / a$ is Hubble parameter. This is a complex equation; to describe cosmological aspects, we must study some limiting cases or specify $\alpha(\phi), V(\phi)$, and $\phi$.
Equation (15) shows the condition for an accelerating universe depending on the choice of nonminimal coupling and the scalar field potential. Hence we determine the following nonminimal coupling:

$$
\alpha(\phi)=\frac{1}{2}\left(1-\xi \phi^{2}\right) \text {. }
$$

For an accelerating universe we should have $\rho_{m}+3 p_{m}<0$ $[12,34]$. If we take $V>0$, we obtain

$$
(1-3 \xi) \dot{\phi}^{2}-V(\phi)-3 \xi \phi(\ddot{\phi}+H \dot{\phi})<0 .
$$

Dynamics of this scalar field are given as

$$
\ddot{\phi}+3 H \dot{\phi}+\xi R \phi+\frac{d V}{d \phi}=0
$$

We obtain

$$
(1-3 \xi) \dot{\phi}^{2}-V(\phi)+3 \xi^{2} R \phi^{2}+6 \xi H \phi \dot{\phi}+3 \xi \phi \frac{d V}{d \phi}<0 .
$$

With $\rho_{m}$ in this case, we determine

$$
\begin{aligned}
y \equiv & \left(1-\xi \phi^{2}\right) \rho_{m}-2 V(\phi) \\
& +\dot{\phi}^{2}\left(\frac{1}{2}-3 \xi\right)+3 \xi^{2} R \phi^{2}+3 \xi \phi \frac{d V}{d \phi}<0,
\end{aligned}
$$

that is, the requirement to have an accelerated universe. In the following, we study the weak energy condition $\rho_{m} \geq 0$ and limit our consideration to the case by $\xi \leq 1 / 6$. Therefore we obtain

$$
-2 V+3 \xi \phi \frac{d V}{d \phi}<y<0,
$$

and a main condition for cosmic acceleration is

$$
V-\frac{3 \xi}{2} \phi \frac{d V}{d \phi}>0, \quad \xi \leq \frac{1}{6} .
$$

For the model to have a cosmic acceleration by $\xi>0$, the potential $V(\phi)$ must change with $\phi$ slower than power-law potential $V_{c}(\phi)=V_{0}\left(\phi / \phi_{0}\right)^{2 / 3 \xi}$ (Figure 1). In other hand, when $\xi<0$, the main condition for cosmic acceleration needs $V$ to grow faster than $V_{c}$ as $\phi$ increases [34]. Now we take a simple special example to show how this model processes. By potential of the form $V(\phi)=\lambda \phi^{n}$, condition (22) takes $\lambda(1-3 n \xi / 2)>0$. In this case just for $\xi \leq 2 / 3 n$ we have the accelerated expansion.

More general case is $\eta \neq 0$ in (13) and (14). In the following we convert the generalized Friedmann equation as

$$
\begin{aligned}
\dot{\chi}+4 & \frac{\dot{a}}{a}\left[\chi+\frac{1}{12 \alpha}\left(\frac{1}{2}(1-2 \xi) \dot{\phi}^{2}-V(\phi)-2 \xi \phi\left(\ddot{\phi}+3 \frac{\dot{a}}{a} \dot{\phi}\right)\right)\right] \\
& =0,
\end{aligned}
$$




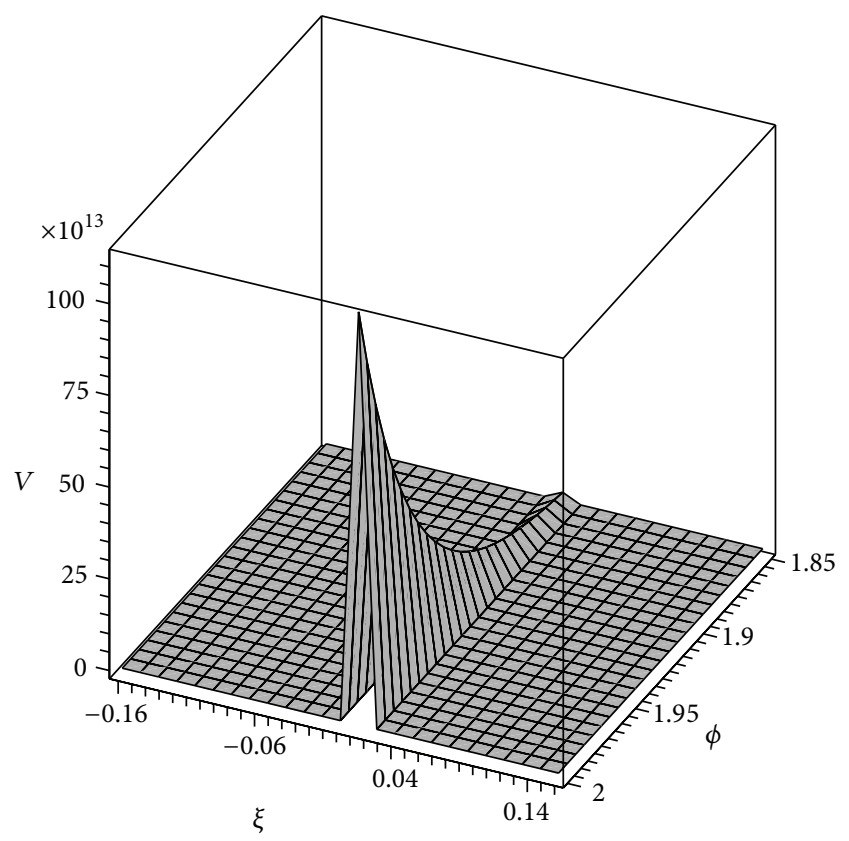

FIgURE 1: $V$ vary for different values of the $\phi$ and nonminimal coupling parameter $\xi$ for $V=\phi^{2 / 3 \xi}$.

where $\chi=(\dot{a} / a)^{2}$ is dark energy field. Then we take $\phi(t) \approx$ $A / t^{\beta}$ where $A$ is a constant and $\beta$ an unspecified power will be studied. For scale factor we take the ansatz $a(t) \approx B t^{\nu}$ where $B$ is a constant and $\nu$ is to be determined. For scalar field potential we take $V(\phi)=\lambda\left(\phi^{2} / 2\right)$. Therefore we obtain

$$
\begin{aligned}
\frac{\ddot{a}}{a}+\left(\frac{\dot{a}}{a}\right)^{2} \approx & \frac{\sigma^{2}}{12 \alpha u}+\frac{\sigma}{24 \alpha u} \rho(1-3 w)+\frac{1}{6 \alpha}(V(\phi)+\Lambda) \\
& -\frac{\dot{\phi}^{2}}{12 \alpha}\left(1+4 \alpha^{\prime \prime}\right)-\frac{\alpha^{\prime}}{3 \alpha}\left(\ddot{\phi}+3 \frac{\dot{a}}{a} \dot{\phi}\right), \\
\ddot{\phi}+3 \frac{\dot{a}}{a} \dot{\phi}+\frac{u^{\prime}}{2 u} \dot{\phi}^{2} \approx & -\frac{6 \alpha}{u} V^{\prime}+10 \frac{\alpha^{\prime}}{u}(V(\phi)+\Lambda) \\
& +4 \frac{\alpha^{\prime}\left(u+\alpha^{\prime} u^{\prime}\right) \sigma}{u^{3}} \rho(1-3 w) \\
& +\frac{8 \alpha^{\prime}\left(u+\alpha^{\prime} u^{\prime}\right)}{u^{3}} \sigma^{2},
\end{aligned}
$$

where $u=6\left[1-\xi(1-(16 / 3) \xi)\left(\phi^{2} / 2\right)\right]$. We take that usual matter on the brane has an equation of state such as $\rho=$ $D t^{-3 v(1+w)}$. Hence we have

$$
\begin{aligned}
& \frac{v(2 \nu-1)}{t^{2}} \\
& \approx \frac{1}{24}\left\{\frac{\sigma^{2}}{3}+4 \Lambda+\left(\frac{\sigma(1-3 w) D}{6}\right) \frac{1}{t^{3 v(1+w)}}\right.
\end{aligned}
$$

$$
\begin{aligned}
+\frac{A^{2}}{t^{2} \beta}[ & 2 \lambda+2 \xi\left(\Lambda+\frac{4(3 / 8-\xi)}{9} \sigma^{2}\right) \\
& +\frac{4 \xi D(1-3 w)(3 / 8-\xi) \sigma}{9} \times \frac{1}{t^{3 \nu(1+w)}} \frac{2 \beta}{t^{2}} \\
& \times(\beta(1-8 \xi)+4 \xi(3 v-1))]\},
\end{aligned}
$$

and scalar field equation is given as

$$
\begin{aligned}
\beta(\beta+1 & -3 \nu) \frac{A}{t^{\beta+2}} \\
\approx & -\frac{\lambda A}{t^{\beta}}\left[1-\frac{8}{3} \xi^{2} \frac{A^{2}}{t^{2 \beta}}\right] \\
& -\frac{5 \xi}{3} \frac{A}{t^{\beta}}\left[\Lambda+\left(\frac{\lambda}{2}+\frac{\xi}{2}\left(1-\frac{16}{3} \xi\right) \Lambda\right) \frac{A^{2}}{t^{2 \beta}}\right] \\
& -\frac{\xi \sigma}{9}\left(2 \sigma+\frac{D}{t^{2}}(1-3 w)\right) \frac{A}{t^{\beta}} \\
& \times\left[1+\xi\left(1-\frac{16}{3} \xi\right)(1+\xi) \frac{A^{2}}{t^{2 \beta}}\right],
\end{aligned}
$$

where $\beta>1$. Two conditions must be solved numerically to limit the values of $\nu$ and $\beta$ for an accelerating expansion. After some calculations we obtain

$$
\begin{gathered}
-\frac{(1-3 w) \xi \sigma}{9} D=A \beta(\beta+1-3 \nu), \\
\lambda=\frac{-\xi \sigma^{2}}{12},
\end{gathered}
$$

where we passed over terms of order $\mathcal{O}\left(t^{-3 \beta}\right)$ and higher. Now, if we arrange $\lambda$, since $\nu(2 \nu-1)=(\sigma(1-3 w) / 144) D$, we find

$$
\beta=\frac{3 v-1}{2} \pm\left[\frac{(3 v-1)^{2}}{2}-16 \xi \nu(2 v-1)\right]^{1 / 2},
$$

where $\nu=2 / 3(1+w)$. For $\nu<1$ the parameter $\beta$ is real for any value; for $\nu>1$ the exponent $\beta$ is real only if we have

$$
\xi \leq \frac{1}{16} \frac{(3 v-1)^{2}}{2 v(2 v-1)} .
$$

These equations limited the values of nonminimal coupling $\xi$ to obtain an accelerating expansion (Figure 2). Hence, it seems that nonminimally coupled scalar field identified on the brane world supplies natural candidate for late-time expansion.

Also we can use (11) to determine dynamical equation of state $\omega(t)$.

Figure 3 shows possible crossing of phantom divided barrier in this framework; this result is important because previous considerations have shown that crossing phantom divided line with a scalar field nonminimally coupled to gravity can exist $[35,36]$. 


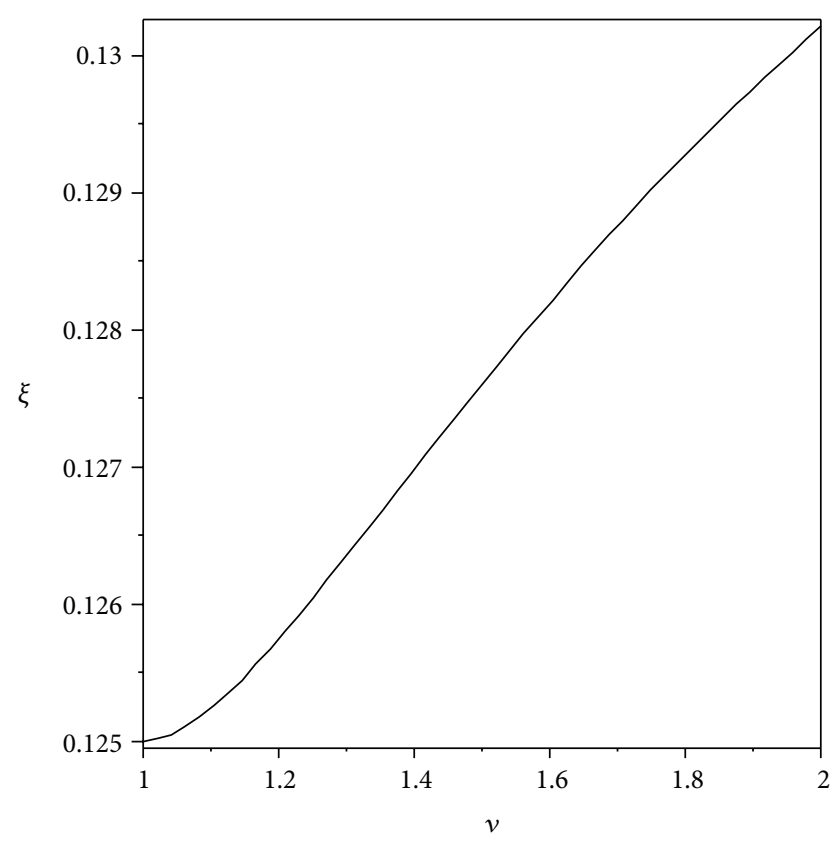

(a)

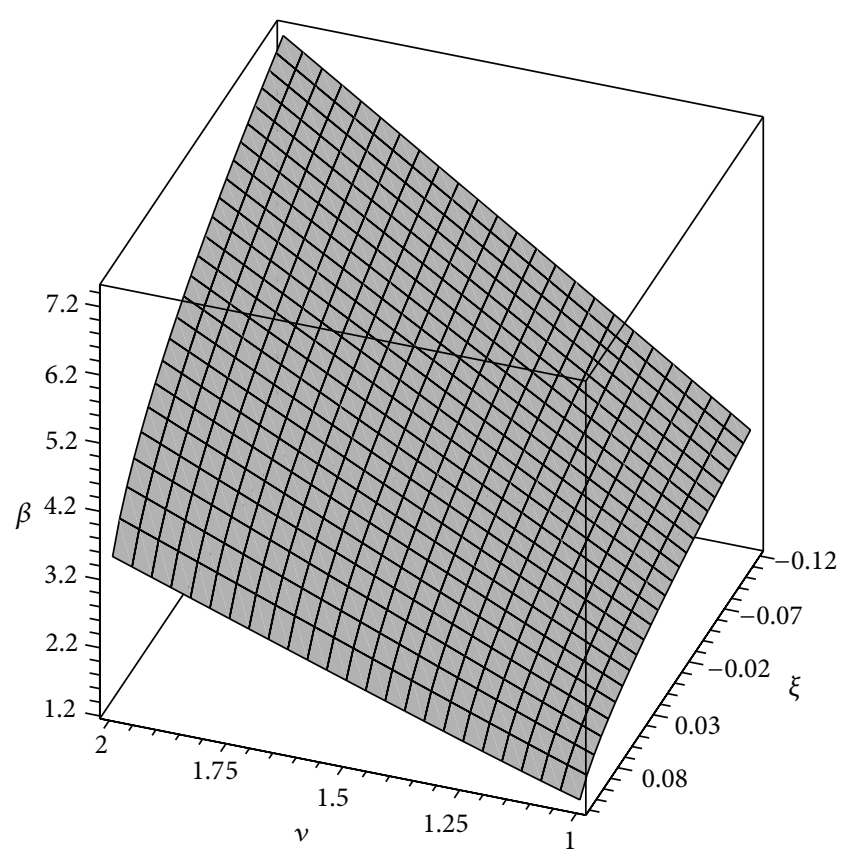

(b)

FIGURE 2: Variation of $\xi$ with respect to $\nu$ with (29) (a) and variation of $\beta$ with respect to $\nu$ and $\xi$ with (28) by sign + , for sign - in (28) $\beta$ has negative value. We choose special range of parameter $\xi$ with (29) in (b) figure.

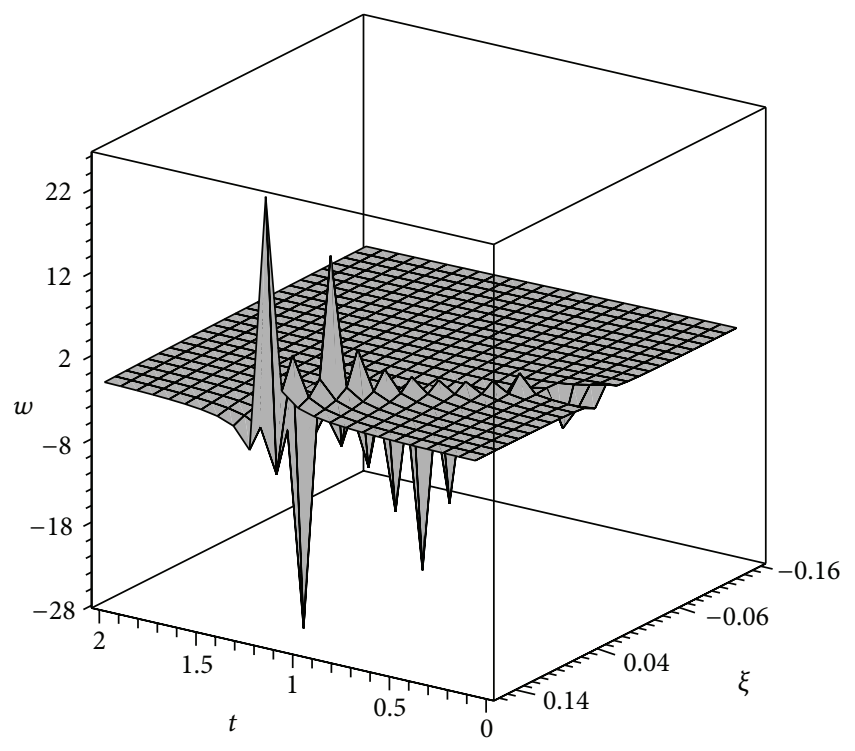

FIGURE 3: Dynamical equation of state $\omega(t)$ varies for different values of the $t$ and nonminimal coupling parameter $\xi$ by (11) and specific choosing for parameters space.

In the following, we explain the physical reasons why the crossing of the phantom divide can occur in this model. Here, we have a scalar field that is nonminimally coupled in gravity and an ordinary matter. Generally, according to [35], these sources of energy momentum can switch together and describe evolution of universe. In this regard, a promising candidate for the dark matter is either a positive cosmological constant or a slowly evolving scalar known as "quintessence." Also the quintessence can be considered as an alternative method to resolve the cosmological constant problem. This is possible because instead of the fine tuning, the quintessence provides a model of slowly decaying cosmological constant. However, there exists another question for the present-day quintessence. This is to ask whether the expansion will keep accelerating forever or it will decelerate again after some time. This is very similar to the exit problem in the inflationary cosmology. According to the theory of quintessence, the dark energy of the universe is dominated by the potential of a scalar field $\phi$ which is still rolling to its minimum at $V=0$.

2.2. Rip Singularity. The additional interest to the models with the crossing phantom divide $\omega<-1$ is caused by their prediction of a Rip singularity [37]. Theoretically, the scale factor of the universe becomes infinite at a finite time in the future which was dubbed Big Rip singularity. There were proposed several scenarios to cure the Big Rip singularity: (I) to consider phantom acceleration as transient phenomenon. This is possible for a number of scalar potentials; (II) to account for quantum effects which may delay/stop the singularity occurrence [38]; (III) to modify the gravitation itself in such a way that it appears to be observationallyfriendly from one side but it cures singularity; (IV) To couple dark energy with dark matter in the special way or to use special (artificial) form for dark energy equation of state [39]. Note that for quintessence dark energy, other (milder) finite-time singularities may occur. For instance, type II (sudden) singularity or type III singularity occurs with finite scale factor but infinite energy and/or pressure. 


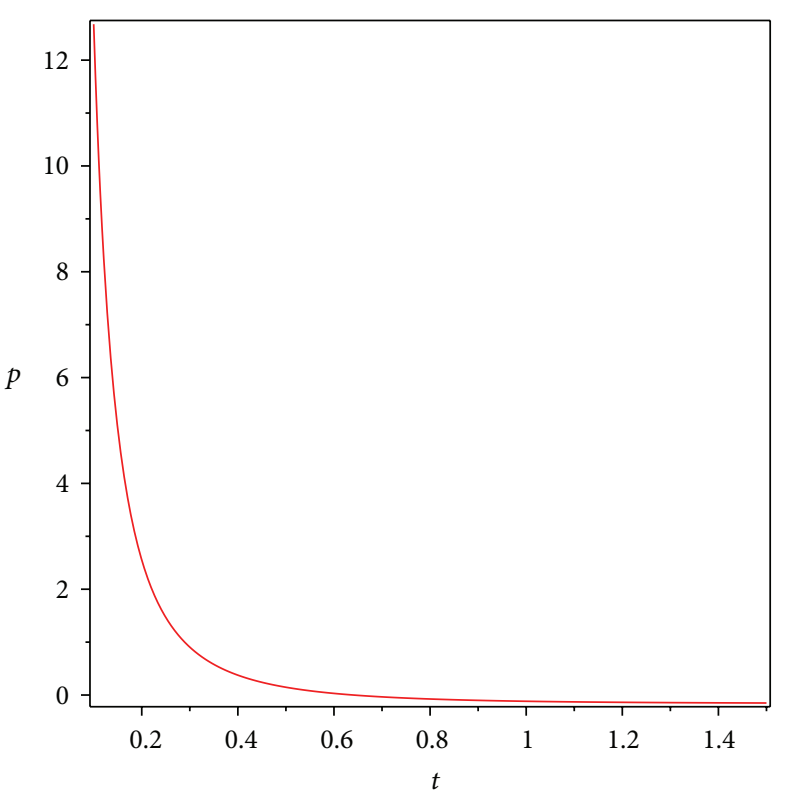

(a)

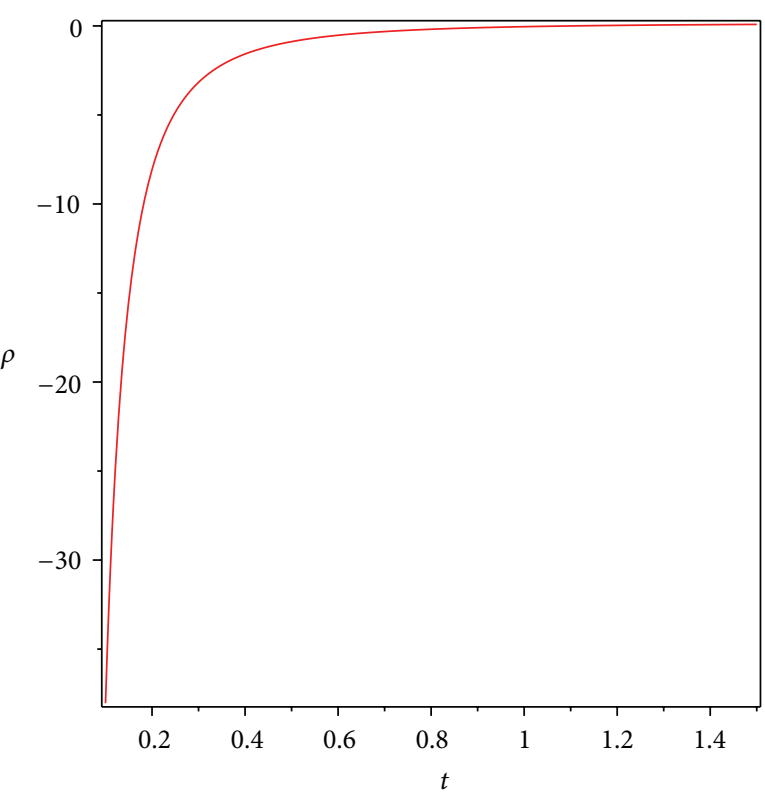

(b)

FIGURE 4: Variation of pressure (a) and energy density (b) with respect to time for a nonminimal coupling model (where $\xi=0.14$ ).

The closer examination shows that the condition $\omega<-1$ is not sufficient for a singularity occurrence [40]. First of all, a transient phantom cosmology is quite possible. Moreover, one can construct such models that $\omega$ asymptotically tends to -1 and the energy density increases with time or remains constant, but there is no finite-time future singularity [40]. Of course, most evident case is when Hubble rate tends to be constant (cosmological constant or asymptotically de Sitter space), which may also correspond to the pseudo rip [31]. Very interesting situation is related with Little Rip cosmology [30] where Hubble rate tends to infinity in the infinite future. The key point is that if $\omega$ approaches -1 sufficiently rapidly, then it is possible to have a model in which the time required for singularity is infinite. However, this position does not appear in our model, because $H$ is finite.

In the following, if we want to consider some types of Rip singularity, we should use Hubble parameter, energy density, and pressure relations that are determined in the previous equations. Therefore, after calculations with $\xi=0.14$ (Figure 4), we obtain results as the following.

Case 1 (type II singularity (sudden)). Conditions: scale factor and energy density reach finite value but $p \rightarrow \infty$.

In this model, we find that scale factor, pressure, and energy density are finite. Therefore, the model does not predict a sudden singularity.

Case 2 (type III singularity). Conditions, scale factor reaches finite value but $(\rho \rightarrow \infty)$ and $|p| \rightarrow \infty$.

This means that the energy density grows so rapidly with time and scale factor does not reach the infinite value. The key difference between Cases 1 and 2 for this model is that the potential of scalar field has a pole in a case of singularity. Here, according to numerical calculations, if scale factor reaches finite value, energy density and pressure are finite too. Hence, type III singularity does not occur in this setup.

Case 3 (pseudo-rip singularity). Conditions: pressure $p \rightarrow$ $-\rho$ in infinite time and leads to $\dot{H} \rightarrow 0$.

This means that the expansion of the universe asymptotically approaches the exponential regime. Obviously in our model with the previous setup, pseudo-rip singularity predict, because in infinite time, when $\dot{H}$ reach to zero, $p \rightarrow$ $-\rho$.

\section{A Lorentz Violating Model}

In this section with using $[13,14,41]$, we consider the cosmological aspects of Lorentz invariance violating model. We emphasize that these model parameters are tightly bounded by both astronomical and cosmological experiments; some works such as $[14,42-44]$ explained that the model parameters are able to be bounded by comparing the model with cosmological observations. Also a global analysis and a group of canonical values for the model parameters to constrain these parameters are given in [45], so that the model is expected to be viable and background evolution is reasonable.

We want to obtain a connection between Lorentz Invariance violation parameter and dynamics of scalar field. First we define an action for a representative scalar-vector-tensor theory which allows Lorentz invariance violation as

$$
S=S_{g}+S_{u}+S_{\phi}
$$


where the actions for the vector field $S_{u}$, the tensor field $S_{g}$, and the scalar field $S_{\phi}$ are assumed as

$$
\begin{aligned}
& S_{g}=\int d^{4} x \sqrt{-g} \frac{1}{16 \pi G} R, \\
& \begin{aligned}
S_{u}=\int d^{4} x \sqrt{-g}[ & -\beta_{1} \nabla^{\mu} u^{\nu} \nabla_{\mu} u_{\nu}-\beta_{2} \nabla^{\mu} u^{\nu} \nabla_{\nu} u_{\mu} \\
& -\beta_{3}\left(\nabla_{\mu} u^{\mu}\right)^{2}-\beta_{4} u^{\mu} u^{\nu} \nabla_{\mu} u^{\alpha} \nabla_{\nu} u_{\alpha} \\
& \left.+\lambda\left(u^{\mu} u_{\mu}+1\right)\right]
\end{aligned} \\
& S_{\phi}=\int d^{4} x \sqrt{-g} \mathscr{L}_{\phi} .
\end{aligned}
$$

This action has a nongravitational degrees of freedom in the structure of Lorentz violating scalar-tensor-vector theory. We take $u^{\mu} u_{\mu}=-1$ and the expected value of vector field $u^{\mu}$ is $\left\langle 0\left|u^{\mu} u_{\mu}\right| 0\right\rangle=-1[45] . \beta_{i}(\phi)(i=1,2,3,4)$ are unrestricted parameters by dimension of mass squared, and also $\mathscr{L}_{\phi}$ is the Lagrangian density for the scalar field in this model $[13,45]$. If universe is homogeneous and isotropic, we consider the universe by metric as

$$
d s^{2}=-\mathscr{N}^{2}(t) d t^{2}+e^{2 \alpha(t)} \delta_{i j} d x^{i} d x^{j},
$$

where $\mathcal{N}$ is a lapse function and the universe scale is given by $\alpha[13,14]$. If the action varies with respect to metric and selecting a fitting gauge, field equations are obtained as

$$
R_{\mu \nu}-\frac{1}{2} g_{\mu \nu} R=8 \pi G T_{\mu \nu}
$$

where $T_{\mu \nu}=T_{\mu \nu}^{(u)}+T_{\mu \nu}^{(\phi)}$ is the total energy-momentum tensor, and energy-momentum tensors of vector and scalar fields are given by $T_{\mu \nu}^{(\phi)}$. The time and space elements of the total energymomentum tensor are obtained as [14]

$$
T_{0}^{0}=-\rho_{u}-\rho_{\phi}, \quad T_{i}^{i}=p_{u}+p_{\phi},
$$

where the energy density and pressure of the vector field are determined as

$$
\begin{gathered}
\rho_{u}=-3 \beta H^{2}, \\
p_{u}=\left(3+2 \frac{H^{\prime}}{H}+2 \frac{\beta^{\prime}}{\beta}\right) \beta H^{2}, \\
\beta \equiv \beta_{1}+3 \beta_{2}+\beta_{3},
\end{gathered}
$$

a prime point to the derivative of any parameters with respect to $\alpha$ and $H \equiv d \alpha / d t=\dot{\alpha}$ is the Hubble parameter. The energy equations for scalar field $\phi$ and the vector field $u$ are given as

$$
\begin{array}{r}
\rho_{u}^{\prime}+3\left(\rho_{u}+p_{u}\right)=+3 H^{2} \beta^{\prime}, \\
\rho_{\phi}^{\prime}+3\left(\rho_{\phi}+p_{\phi}\right)=-3 H^{2} \beta^{\prime} .
\end{array}
$$

So, the total energy equation for the vector and the scalar fields is written as

$$
\rho^{\prime}+3(\rho+p)=0, \quad\left(\rho=\rho_{u}+\rho_{\phi}\right) .
$$

Now, dynamics of the model are determined with the following Friedmann equations [13, 14]:

$$
\begin{gathered}
\left(1+\frac{1}{8 \pi G \beta}\right) H^{2}=\frac{1}{3 \beta} \rho_{\phi}, \\
\left(1+\frac{1}{8 \pi G \beta}\right)\left(H H^{\prime}+H^{2}\right)=-\frac{1}{6}\left(\frac{\rho_{\phi}}{\beta}+\frac{3 p_{\phi}}{\beta}\right)-H^{2} \frac{\beta^{\prime}}{\beta} .
\end{gathered}
$$

For the scalar section of this model we assume the following Lagrangian:

$$
\mathscr{L}_{\phi}=-\frac{\eta}{2}(\nabla \phi)^{2}-V(\phi),
$$

where $(\nabla \phi)^{2}=g^{\mu \nu} \partial_{\mu} \phi \partial_{\nu} \phi$. Usual scalar fields are matched to $\eta=1$ while $\eta=-1$ describes phantom fields. The homogeneous scalar field has the density $\rho_{\phi}$ and pressure $p_{\phi}$ as

$$
\begin{aligned}
& \rho_{\phi}=\frac{\eta}{2} H^{2} \phi^{\prime 2}+V(\phi), \\
& p_{\phi}=\frac{\eta}{2} H^{2} \phi^{\prime 2}-V(\phi),
\end{aligned}
$$

and equation of state parameter is obtained as

$$
\omega_{\phi}=\frac{p_{\phi}}{\rho_{\phi}}=-\frac{1-\eta H^{2} \phi^{\prime 2} / 2 V}{1+\eta H^{2} \phi^{\prime 2} / 2 V} .
$$

Now the Friedmann equation takes a form as [14]

$$
H^{2}=\frac{1}{3 \bar{\beta}}\left[\frac{\eta}{2} H^{2} \phi^{\prime 2}+V(\phi)\right],
$$

where $\bar{\beta}=\beta+1 / 8 \pi G$. With this equation we obtain

$$
\phi^{\prime}=-2 \eta \bar{\beta}\left(\frac{H_{, \phi}}{H}+\frac{\bar{\beta}_{, \phi}}{\bar{\beta}}\right) .
$$

If this equation is substituted into the Friedmann equation, we can find the potential of the scalar field as [14]

$$
V=3 \bar{\beta} H^{2}\left[1-\frac{2}{3} \eta \bar{\beta}\left(\frac{\bar{\beta}_{, \phi}}{\bar{\beta}}+\frac{H_{, \phi}}{H}\right)^{2}\right],
$$

where $H=H(\phi(t))$. The equation of state is found in following form:

$$
\begin{aligned}
\omega_{\phi} & =-1+\frac{4}{3} \eta \bar{\beta}\left(\frac{H_{, \phi}}{H}+\frac{\bar{\beta}_{, \phi}}{\bar{\beta}}\right)^{2} \\
& =-1+\frac{1}{3} \eta \frac{\phi^{\prime 2}}{\bar{\beta}} .
\end{aligned}
$$

Relations (43) and (45) have main role in following considerations. We can see violation of the Lorentz invariance which 
has been described by existence of a vector field in the action; now it has been combined in the dynamics of scalar field and equation of state by $\bar{\beta}$ parameter. This situation allows us to consider phantom divided line crossing in LIV model. Now we should solve (43) and (45), to obtain dynamics of scalar field $\phi$ and the equation of state $\omega_{\phi}$. Therefore, we must define the Hubble parameter $H(\phi)$ and the vector field, $\bar{\beta}(\phi)$. In the following, we choose a general case of the Hubble parameter $H(\phi)$ and the vector field $\bar{\beta}(\phi)$ and then consider crossing of phantom divided line, late-time acceleration, and Rip singularity scenario.

3.1. Considering Late-Time Acceleration in LIV Model. For an acceleration universe that should be $\ddot{a}>0$, we rewrite it to this form $H^{\prime} / H>-1$. To consider this case, we must determine some equations. We study a general case for the vector field and the Hubble parameter; note that these equations are a function of $\phi[46]$

$$
H=H_{0} \phi^{\zeta}, \quad \bar{\beta}(\phi)=m \phi^{n}, \quad n>2 .
$$

Following, we just study a quintessence scalar field by $\eta=1$. By using relation (43), we calculate

$$
\phi(t)=\frac{1}{A},
$$

where

$$
\begin{aligned}
A=\left[H_{0}\left(t-t_{0}\right)\right. & \left(-4 \zeta m+4 \zeta m n+2 \zeta^{2} m\right. \\
& \left.\left.-4 m n+2 m n^{2}\right)+\phi_{0}\right]^{(1 /(n+\zeta-2))},
\end{aligned}
$$

and by using relation (45), we can obtain

$$
\omega_{\phi}(t)=-1+\frac{4}{3} m \phi^{n-2}(t)(\zeta+n)^{2} .
$$

Following these relations (46), (45), and (49), we find

$$
m^{2}<\frac{1}{4(-1)^{n} \phi^{n-2}(t)(\zeta+n)^{2}}, \quad n>2 .
$$

This equation describes a restriction in LIV parameters for a late-time acceleration.

Now with using (46) and (47) to obtain scale factor as

$$
\begin{aligned}
& a(t)=a_{0}\left(t_{0}\right) \\
& \times e^{\left(2 \phi_{0}^{\zeta /(n+\zeta-2)}\left(m(n+\zeta)(n+\zeta-2) H_{0}\left(t-t_{0}\right)+(1 / 2) \phi_{0}\right) e^{F}-\phi_{0}\right) / 2 \phi_{0}^{\zeta /(n+\zeta-2)}(n-2) m(n+\zeta),}
\end{aligned}
$$

where

$$
F=\zeta \ln \left(\frac{1}{e^{\ln \left(2 m(n+\zeta)(n+\zeta-2) H_{0}\left(t-t_{0}\right)+\phi_{0}\right) /(n+\zeta-2)}}\right),
$$

we should select a special space parameter; therefore we approach (51) by a Taylor series in special space parameter

$$
a(t)=.286504+.447663 t+.237821 t^{2}+.0352651 t^{3} .
$$

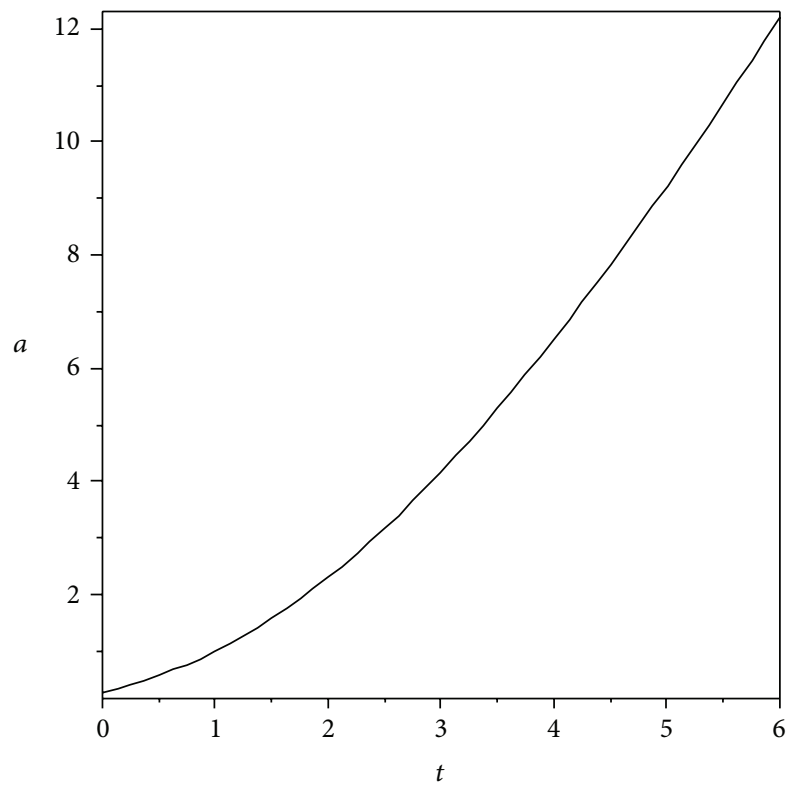

FIgURE 5: Variation of scale factor $a(t)$ for different values of $t$ for $n=3, \zeta=-2$ and $m=-0.1$. The values of $\zeta$ are determined by relation (50).

We can see in Figure 5 acceleration evolution in late time. Now, we study equation of state to determine crossing phantom divided line. By $\phi$ according to (47), the equation of state is obtained as

$$
\omega_{\phi}(t)=-1+\frac{4}{3} m \frac{(\zeta+n)^{2}}{E},
$$

where

$$
\begin{aligned}
E=[ & H_{0}\left(t-t_{0}\right)\left(-4 \zeta m+4 \zeta m n+2 \zeta^{2} m-4 m n+2 m n^{2}\right) \\
& \left.+\phi_{0}\right]^{((n-2) /(n+\zeta-2))},
\end{aligned}
$$

which implicitly has a dynamical behavior. This model lets us choose a suitable parameter space to crossing phantom divided line. Moreover, this parameter space must be compared by observational data. We insist in this model that a scalar field and a vector field together can explain crossing phantom divided barrier and late-time acceleration. We can see in Figure 6 the crossing of phantom divided line for a dynamical equation of state. Figure 6 perhaps explains why we are living in an era of $\omega<-1$. Note that $\bar{\beta}(t)$ has the main role of Lorentz invariance violation model. The dynamical equation for $\bar{\beta}(t)$ has an interested mean: with a suitable fine tuning we can obtain a Lorentz violating cosmology correspond to observational data.

We know many different models that explain phantom divided line crossing, but this model is special because it contains only a scalar field and a Lorentz violating vector field that checks the crossing [47-49]. However, two notices must be explained in this paper: first, in Figures 3 and 6 we can see that there are some sudden jerks of the equation 


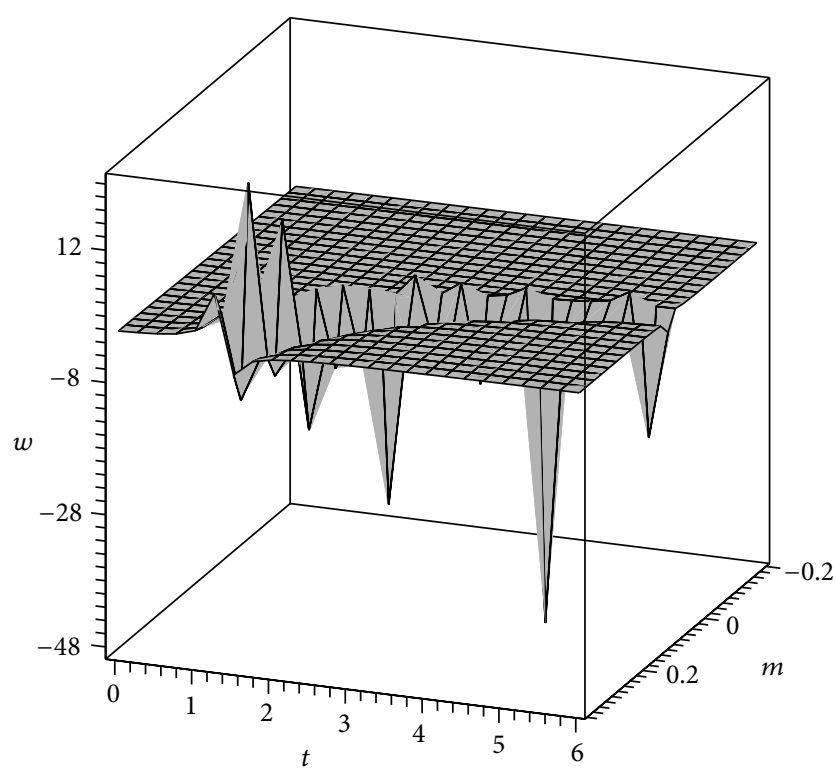

FIGURE 6: $\omega_{\phi}$ vary for different values of the vector field by $m$ and $t$ for $n=3$ and $\zeta=-2$. Positive values of $\zeta$ do not show a phantom divided line crossing. The values of $\zeta$ are obtained with (50).

of state. In some models that equation of state crossing the phantom divided line, $\omega$ surge around -1 (see [50] and references therein). These jerks are certainly a signature of chaotic manner of equation of state. Second, in those figures we see that crossing of the phantom divided line appears at late time. This means that second cosmological coincidence problem requires extra fine tuning in model parameters.

For describing the physical reasons why the crossing of the phantom divide can occur in this model, we can state by a suitable coupling between a quintessence scalar fields and other matter content can lead to a constant ratio of the energy densities of both components which is compatible with an accelerated expansion of the universe or crossing of phantom divide line. In our model, we have three sources of energy momentum: (1) standard ordinary matter, (2) scalar field as a candidate of dark energy, and (3) energy-momentum content depending on Lorentz violating vector field. Here we assume that standard matter has negligible contribution on the total energy-momentum content of the universe. For other two energy-momentum contents, it is possible to use the "trigger mechanism" to explain dynamical equation of state. This means that we assume that scalar-vector-tensor theory contains Lorentz invariance violation which acts like the hybrid inflation models. In this situation, vector and scaler fields play the roles of inflaton and the "waterfall" fields, respectively. Therefore it is reasonable to expect that one of them will eventually dominate to explain inflation or accelerating phase and crossing of phantom divided line.

3.2. Rip Singularity. According to Rip singularity scenario that is explained in previous Section 2.2, we consider some types of Rip singularity in this LIV model. As shown in Figures 5 and 6 , we provide suitable conditions for considering
Rip singularity scenario. Hence, corresponding to equation of state and using equations such as energy density, pressure, and Hubble parameter, we can study some Rip singularity solutions.

According our setup in this paper that selected $\zeta=-2$ and $n=3$ in anzats (46) we have

$$
H=H_{0} \phi^{-2}, \quad \beta=m \phi^{3} .
$$

Now with substituting previous equations in energy density and pressure relations, we can consider dynamical behaviors in these relations. Note that we selected for ordinary matter $\rho_{m}=0$ and $p_{m}=0$. Now after numerical calculations (Figure 7), we obtain results as the following.

In finite time, scale factor and energy density increase, but pressure decreases. Therefore type II singularity (sudden) does not predict. But type III singularity appears because $|p|$ decrease so rapidly. In infinite time, Hubble rate tends to zero; therefore Little Rip singularity is impossible, but pseudorip singularity can occur. We emphasize this relations to determine original setup in this paper. This means by different selection in parameters of $\zeta$ and $m$, all dynamics of relations change. Therefore, one can change setup and consider other options. However, in this situation, our work is limited.

\section{Comparison with Observational Data and Other Dark Energy Models}

4.1. Analyzing SNe Data. The parameters of the cosmological models can be determined from a strict comparison of their predictions with accurate observational data. Here we consider the data coming from $\mathrm{SNe}$ observations, the evolution of the Hubble parameter in the brane model.

The modulus $\mu$ versus redshift $z=a_{0} / a-1$ relation corresponding to type Ia supernovae from the Supernova Cosmology Project ([51], Uni 2013 [52]) is, as well known,

$$
\mu(z)=\mu_{0}+5 \lg D_{L}(z) .
$$

The relation for the luminosity distance $D_{L}(z)$ as a function of the redshift in the FRW cosmology (FC) is

$$
D_{L}^{\mathrm{FC}}=\frac{c}{H_{0}}(1+z) \int_{0}^{z} h^{-1}(z) d z,
$$

where $h(z)=\left[\Omega_{m 0}(1+z)^{3}+\Omega_{D 0} F(z)\right]^{1 / 2}$.

Here, $\Omega_{m 0}$ is the total fraction of matter density, $\Omega_{D 0}$ is the fraction of $D E$ energy density, and $H_{0}$ is the current Hubble parameter. The constant value $\mu_{0}$ depends on the chosen Hubble parameter:

$$
\mu_{0}=42.384-5 \log h, \quad h=H_{0} / 100 \mathrm{~km} / \mathrm{s} / \mathrm{Mpc} .
$$

The function $F(z)=\rho_{D}(z) / \rho_{D 0}$ can be determined from the continuity equation

$$
\dot{\rho}_{D}-3 \frac{\dot{a}}{a} g\left(\rho_{D}\right)=0,
$$

which can be rewritten as

$$
\int_{\rho_{\mathrm{D} 0}}^{\rho_{D}(z)} \frac{d y}{g(y)}=-3 \ln (1+z)
$$




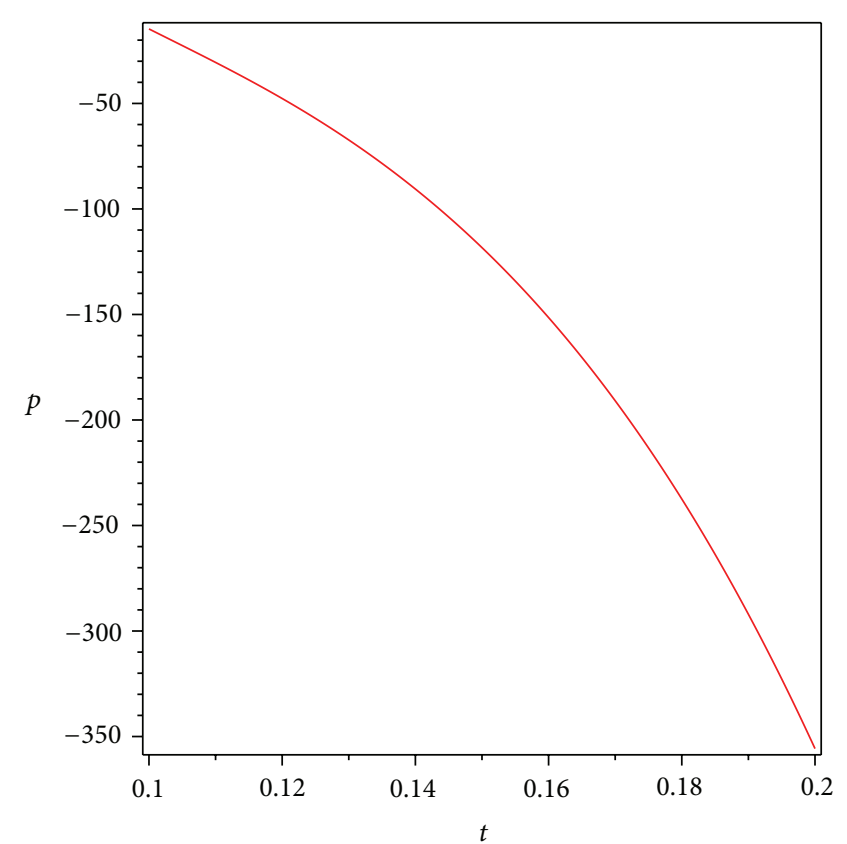

(a)

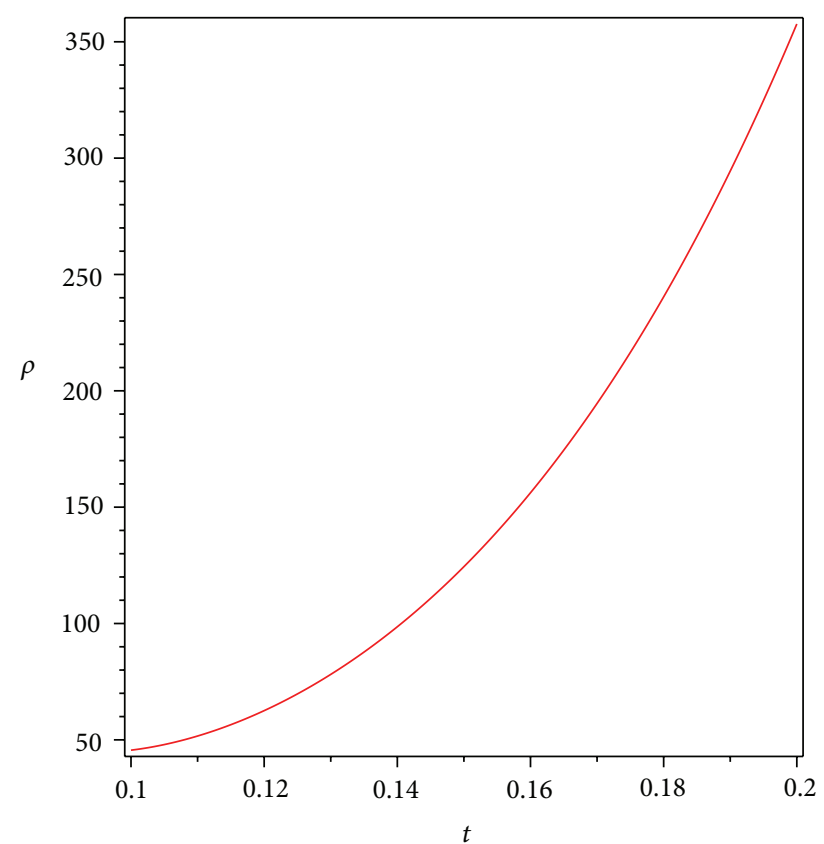

(b)

FIGURE 7: Variation of pressure (a) and energy density (b) with respect to time for a minimal coupling model (where $\zeta=-2$ and $n=3$ ).

For simplicity, we neglect the contribution of radiation. For cosmology on the brane (BC), (58) can be rewritten as

$$
D_{L}^{\mathrm{BC}}=\frac{c}{H_{0}}(1+z) \int_{0}^{z} h^{-1}(z)\left[1+\delta h^{2}(z)\right]^{-1 / 2}(1+\delta)^{1 / 2} d z
$$

where for convenience the parameter $\delta=\rho_{0} / 2$ has been introduced. For the analysis of the $\mathrm{SNe}$ data one needs to calculate the parameter $\chi^{2}$, which is defined by

$$
\chi_{\mathrm{SN}}^{2}=\sum_{i} \frac{\left[\mu_{\mathrm{obs}}\left(z_{i}\right)-\mu_{t h}\left(z_{i}\right)\right]^{2}}{\sigma_{i}^{2}}
$$

where $\sigma_{i}$ is the corresponding $1 \sigma$ error. The parameter $\mu_{0}$ is independent of the data points, and, therefore, one has to perform a uniform marginalization over $\mu_{0}$. Minimization with respect to $\mu_{0}$ can be done by simply expanding the $\chi_{\mathrm{SN}}^{2}$ with respect to $\mu_{0}$,

$$
\chi_{\mathrm{SN}}^{2}=A-2 \mu_{0} B+\mu_{0}^{2} C,
$$

where

$$
\begin{gathered}
A=\sum_{i} \frac{\left[\mu_{\mathrm{obs}}\left(z_{i}\right)-\mu_{t h}\left(z_{i} ; \mu_{0}=0\right)\right]^{2}}{\sigma_{i}^{2}}, \\
B=\sum_{i} \frac{\mu_{\mathrm{obs}}\left(z_{i}\right)-\mu_{t h}\left(z_{i}\right)}{\sigma_{i}^{2}}, \quad C=\sum_{i} \frac{1}{\sigma_{i}^{2}} .
\end{gathered}
$$

The expression has a minimum for $\mu_{0}=B / C$ at

$$
\bar{\chi}_{\mathrm{SN}}^{2}=A-\frac{B^{2}}{C} \text {. }
$$

TABLE 1: Hubble parameter versus redshift data.

\begin{tabular}{lcc}
\hline$z$ & $\begin{array}{c}H_{\mathrm{obs}}(z) \\
\mathrm{km} \mathrm{s}^{-1} \mathrm{Mpc}^{-1}\end{array}$ & $\begin{array}{c}\sigma_{H} \\
\mathrm{~km} \mathrm{~s}^{-1} \mathrm{Mpc}^{-1}\end{array}$ \\
\hline 0.170 & 83 & 8 \\
1.530 & 140 & 14 \\
1.750 & 202 & 40 \\
\hline
\end{tabular}

One can minimize $\bar{\chi}_{\mathrm{SN}}^{2}$ instead of $\chi_{\mathrm{SN}}^{2}$. With [53] and Table 1, one determines the $56.01 \%$ confidence level by $\Delta \chi^{2}=\chi^{2}-$ $\chi_{\min }^{2}<1.01$ for the one-parameter or 1.9 for the twoparameter model. Similarly, the $91.2 \%$ confidence level is determined by $\Delta \chi^{2}=\chi^{2}-\chi_{\min }^{2}<3.78$ or 5.63 for the oneand two-parameter models, respectively.

4.2. Dark Energy Cosmology. In this paper, we consider two models that explain dark energy in content of the universe, but according to [54] and references therein, there are many different models in this subject. Therefore, in this section for a worthy review, we briefly point out them.

As we know a number of popular dark energy models, such as the $\Lambda C D M$ model, Little Rip and Pseudo-Rip, scenarios, the phantom and quintessence cosmologies with the four types (I, II, III, and IV) of the finite-time future singularities and nonsingular universes filled with dark energy have been studied.

Investigations show the $\Lambda \mathrm{CDM}$ model and recent various cosmological observations to give the bounds on the latetime acceleration of the universe. Furthermore, researchers have considered a fluid description of the universe in which 
the dark fluid has a general form of the EoS covering the inhomogeneous and imperfect EoS. They have shown that all the dark energy cosmologies can be realized by different fluids and also considered their properties. It has also been demonstrated that at the current stage the cosmological evolutions of all the dark energy universes may be similar to those of the $\Lambda \mathrm{CDM}$ model, and hence these models are compatible with the cosmological observations. In other hand, they have studied the equivalence of different dark energy models, that is, single and multiple scalar field theories, tachyon, scalar theory and holographic dark energy, in which the quintessence/phantom cosmology with the current cosmic acceleration can be represented, and eventually verified those equivalence to the corresponding fluid descriptions. In this regards, as another equivalent class of dark energy models, in which dark energy has its geometrical origins, namely, modifications of gravitational theories, one has examined $F(R)$ gravity including its extension to $F(R)$ Horava-Lifshitz gravity and $f(T)$ gravity. It has clearly been explored that in these models, the $\Lambda \mathrm{CDM}$ model or the late-time cosmic acceleration with the quintessence/phantom behavior can be performed. It is shown that all the previous dark energy universes may mimic the $\Lambda \mathrm{CDM}$ model currently, consistent with the recent observational data. Furthermore, special attention is paid to the equivalence of different dark energy models.

However, it is significant to remark that there are a number of various dark energy models such as $F(\mathscr{G})$ gravity, where $\mathscr{G} \equiv R^{2}-4 R_{\mu \nu} R^{\mu \nu}+R_{\mu \nu \rho \sigma} R^{\mu \nu \rho \sigma}$ with $R_{\mu \nu}$ and $R_{\mu \nu \xi \sigma}$ being the Ricci tensor Riemann tensors, respectively, is the GaussBonnet invariant, $F(R, \mathscr{G})$ gravity, scalar-Gauss-Bonnet dark energy, $k$-essence dark energy models, ghost condensates scenario, viscous dark energy, nonminimal derivative dark energy models, $G$-essence dark energy models, nonlocal gravity produced by quantum effect, which is investigated to account for the coincidence problem of dark energy and dark matter, and galilean dark energy models. In particular, galilean gravity has recently been studied very extensively in the literature. The most important feature of the Lagrangian for the galilean scalar field is that the equation of motion derived from the Lagrangian is up to the second order, so that the appearance of an extra degree of freedom with the existence of a ghost can be avoided. The galilean field originates from a brane bending mode in the Dvali-Gabadadze-Porrati (DGP) brane world scenario, and therefore galilean gravity might be regarded as an indirect resolution for the issue of a ghost in the self-accelerating branch of the DGP model. In all of the previous models, in each model, the difference is only the forms of the energy density $\rho_{D E}$ and pressure $P_{D E}$ of dark energy. Hence, the expression of the Hubble parameter $H$ to describe the concrete cosmology, for example, the $\Lambda \mathrm{CDM}$, quintessence, and phantom cosmologies, can be reconstructed by using gravitational field equations. Similarly, by applying $\rho_{D E}$ and $P_{D E}$, the $\operatorname{EoS} w_{D E} \equiv D E / \rho_{D E}$ in the fluid description can also be presented.

Finally, we emphasize the role of cosmography in this discussion. As shown, it is a fundamental tool because it allows, in principle, to discriminate among models without a priori assumptions but just laying on constraints coming from data. However, the main criticism to this approach is related to the extension of the Hubble series, the quality and the richness of data samples. In particular, observations cannot be extended at any redshift and, in most of cases, are not suitable to track models up to early epochs. However, the forthcoming observational campaigns should ameliorate the situation removing the degeneration emerging at low redshifts and allowing a deeper insight of models.

\section{Summary}

Several pieces of evidence from observational data have shown that our universe is in the accelerated expansion era. In this paper, we have shown this reality in two different models: a nonminimally coupling scalar field identified on the brane and a scalar field coupling minimally to gravity in LIV model. According to complex of dynamical equations, we have limited our research to some special form of nonminimal coupling and scalar field potential. Then we have studied special form of time evolution for the scale factor and a scalar field. In brane world model, we have determined an equation that explains situations where an accelerating expansion of the universe is possible. As a consequence, we have obtained which nonminimally coupling scalar field identified on the brane is a suitable candidate for late-time expansion of universe. In LIV model, we have considered a new framework for crossing of phantom divided line with equation of state that combined a violation of Lorentz invariance in a cosmological model. However, we have explained with a suitable choice of parameter space that it is possible to have phantom divided line crossing just with a Lorentz invariance violating vector field and a single scalar field $[35,36]$. In this regard, existence of a Lorentz invariance violating vector field prepares a setup for crossing phantom divided line just with a minimally coupling scalar field. In other view point, there is the possibility of a Rip singularity by suitable tuning in the parameters of models.

Comparison of nonminimal model with WMAP data gives more accurate constraints on the values of nonminimal coupling. A detailed comparison between our results and some results of previous studies shows that our constraints for nonminimal coupling with exponential potential are consistent with holographic dark energy framework and also with the result of warped DGP brane model [35].

\section{Appendices}

\section{A. Proof of Stability}

Now we study the stability of our model. In order to study the classical stability of our model, we analyze the behavior of the model in the $\omega-\omega^{\prime}$ plane where $\omega^{\prime}$ is the derivative of $\omega$ with respect to the logarithm of the scale factor (see [14] for a similar analysis for other interesting cases)

$$
\omega^{\prime} \equiv \frac{d \omega}{d \ln a}=\frac{d \omega}{d t} \frac{d t}{d \ln a}=\frac{\dot{\omega}}{H} .
$$


The sound speed expresses the phase velocity of the inhomogeneous perturbations of the field. Therefore, we define the function $c_{a}$ as

$$
c_{a}^{2} \equiv \frac{\dot{p}}{\dot{\rho}} .
$$

If the matter is considered as a perfect fluid, this function would be the adiabatic sound speed of this fluid. We note that with scalar fields that do not obey perfect fluid form necessarily, this quantity is not actually a sound speed. The conservation of the total (scaler and vector) field is given by (37). As we see, the equation implicitly depended on $\beta$ and $H$ forms. Hence, we can consider anzats (46) with substituting in (37) as

$$
\frac{d \rho_{\text {total }}}{d t}+3\left(\rho_{\text {total }}+p_{\text {total }}\right)=0
$$

Since the dust matter obeys the continuity equation and the Bianchi identity keeps valid, total energy density satisfies the continuity equation. From the previous equation, we have

$$
\dot{\rho}_{d e}=-3 \rho_{d e}\left(1+\omega_{d e}\right),
$$

where de means dark energy. Using equation of state $p_{d e}=$ $\omega_{d e} \rho_{d e}$, we obtain

$$
\dot{p}_{d e}=\dot{\omega} \rho_{d e}+\omega_{d e} \dot{\rho}_{d e} .
$$

Therefore, the function $c_{a}^{2}$ could be rewritten as

$$
c_{a}^{2}=\frac{\dot{\omega}_{d e}}{-3\left(1+\omega_{d e}\right)}+\omega_{d e}
$$

In this situation, with suitable choice of $\zeta$ and $n$ in (46), we obtained that the $\omega-\omega^{\prime}$ plane is divided into four regions defined as follows :

$$
\begin{array}{ll}
\text { I : } \omega_{d e}>-1, & \omega^{\prime}>3 \omega(1+\omega) \Longrightarrow c_{a}^{2}>0, \\
\text { II : } \omega_{d e}>-1, & \omega^{\prime}<3 \omega(1+\omega) \Longrightarrow c_{a}^{2}<0, \\
\text { III : } \omega_{d e}<-1, & \omega^{\prime}>3 \omega(1+\omega) \Longrightarrow c_{a}^{2}<0, \\
\text { IV : } \omega_{d e}<-1, & \omega^{\prime}<3 \omega(1+\omega) \Longrightarrow c_{a}^{2}>0 .
\end{array}
$$

As one can see from these relations, the regions I and IV have the classical stability in our model.

\section{B. Initial Conditions for Numerical Calculations in Both Models}

B.1. In the Brane World Model. In the induced gravity model we must add the additional condition

$$
\rho \gg\left|6 \alpha\left(H^{2}+\frac{K}{a^{2}}\right)\right| \text {. }
$$

Thus we require both high energy and weak coupling. In addition, the last condition and the continuity equation imply that $P=-\rho$ for $H \neq 0$. Note, however, that unlike 4D general relativity, we do not necessarily require $\rho=$ constant. In other hand, initial values for parameters $\xi$ and $\beta$ are obtained from equation

$$
\begin{gathered}
\xi \leq \frac{1}{16} \frac{(3 v-1)^{2}}{2 v(2 v-1)} \\
\beta=\frac{3 v-1}{2} \pm\left[\frac{(3 v-1)^{2}}{2}-16 \xi \nu(2 v-1)\right]^{1 / 2} .
\end{gathered}
$$

B.2. In Lorentz Invariance Violation Model. The dynamical attractor of the cosmological system has been employed to make the late-time behaviors of the model insensitive to the initial condition of the field and thus alleviates the fine tuning problem. In quintessence models, the dynamical system has tracking attractor that makes the quintessence evolves by tracking the equation of state of the background cosmological fluid so as to alleviate the fine tuning problem.

Thus, in order to construct viable Lorentz violation model, we require that the coupling function $\bar{\beta}$ and the potential function $V$ should satisfy the condition

$$
\begin{gathered}
\overline{\beta \beta}_{, \phi \phi} \\
\bar{\beta}_{, \phi}^{2}>\frac{1}{2}, \\
\frac{V V_{, \phi \phi}}{V_{, \phi}^{2}}+\frac{1}{2} \frac{\bar{\beta}_{, \phi} / \bar{\beta}}{V_{, \phi} / V}>1,
\end{gathered}
$$

respectively.

\section{References}

[1] N. Arkani-Hamed, S. Dimopoulos, and G. Dvali, “The hierarchy problem and new dimensions at a millimeter," Physics Letters B, vol. 429, no. 3-4, pp. 263-272, 1998.

[2] L. Randall and R. Sundrum, "Large mass hierarchy from a small extra dimension," Physical Review Letters, vol. 83, no. 17, pp. 3370-3373, 1999.

[3] G. Dvali, G. Gabadadze, and M. Porrati, "4D gravity on a brane in 5D Minkowski space," Physics Letters B, vol. 485, no. 1-3, pp. 208-214, 2000.

[4] P. Binétruy, C. Deffayet, and D. Langlois, "Non-conventional cosmology from a brane universe," Nuclear Physics B, vol. 565, no. 1-2, pp. 269-287, 2000.

[5] P. Binetruy, C. Deffayet, U. Ellwanger, and D. Langloisb, "Brane cosmological evolution in a bulk with cosmological constant," Physics Letters B, vol. 447, pp. 285-291, 2000.

[6] R. Maartens, "Brane-world gravity," Living Reviews in Relativity, vol. 7, p. 7, 2004.

[7] H. Collins and B. Holdom, "Brane cosmologies without orbifolds," Physical Review D, vol. 62, no. 10, Article ID 105009, 7 pages, 2000.

[8] D. Langlois and M. Rodríguez-Martínez, "Brane cosmology with a bulk scalar field," Physical Review D, vol. 64, no. 12, Article ID 123507, 9 pages, 2001.

[9] C. Bogdanos, A. Dimitriadis, and K. Tamvakis, "Brane models with a Ricci-coupled scalar field," Physical Review D, vol. 74, no. 4, Article ID 045003, 11 pages, 2006. 
[10] K. Farakos and P. Pasipoularides, "Second Randall-Sundrum brane world scenario with a nonminimally coupled bulk scalar field," Physical Review D, vol. 73, Article ID 084012, 12 pages, 2006.

[11] S. Mizuno, K. Maeda, and K. Yamamoto, "Dynamics of a scalar field in a brane world," Physical Review D, vol. 67, Article ID 023516, 15 pages, 2003.

[12] M. Bouhamdi-Lopez and D. Wands, "Induced gravity with a nonminimally coupled scalar field on the brane," Physical Review D, vol. 71, no. 2, Article ID 024010, 10 pages, 2005.

[13] S. Kanno and J. Soda, "Lorentz violating inflation," Physical Review D, vol. 74, Article ID 063505, 8 pages, 2006.

[14] Arianto, F. P. Zen, B. E. Gunara, Triyanta, and Supardi, "Some impacts of Lorentz violation on cosmology," Journal of High Energy Physics, vol. 2007, no. 09, article 048, 2007.

[15] S. Perlmutter, G. Aldering, G. Goldhaber et al., "Measurements of $\Omega$ and $\Lambda$ from 42 High-Redshift Supernovae," The Astrophysical Journal, vol. 517, no. 2, p. 565, 1999.

[16] A. G. Riess, A. V. Filippenko, P. Challis et al., "Observational evidence from supernovae for an accelerating universe and a cosmological constant," The Astronomical Journal, vol. 116, no. 3, p. 1009, 1998.

[17] A. D. Miller, R. Caldwell, M. J. Devlin et al., "Measurement of the angular power spectrum of the cosmic microwave background from $l=100$ to 400," The Astrophysical Journal Letters, vol. 524, no. 1, p. L1, 1999.

[18] P. de Bernardis, P. A. R. Ade, J. J. Bock et al., "A flat Universe from high-resolution maps of the cosmic microwave background radiation," Nature, vol. 404, pp. 955-959, 2000.

[19] S. Hanany, P. Ade, A. Balbi et al., "MAXIMA-1: a measurement of the cosmic microwave background anisotropy on angular scales of $10^{\prime}-5^{\circ}$,' The Astrophysical Journal Letters, vol. 545, no. 1, p. L5, 2000.

[20] D. N. Spergel, L. Verde, H. V. Peiris et al., "First-year Wilkinson Microwave Anisotropy Probe (WMAP)* observations: determination of cosmological parameters," The Astrophysical Journal, vol. 148, no. 1, p. 175, 2003.

[21] L. M. Page, R. Nolta, C. Barnes et al., "First-year Wilkinson Microwave Anisotropy Probe (WMAP)* observations: interpretation of the TT and TE angular power spectrum peaks," The Astrophysical Journal, vol. 148, Article ID 377224, p. 233, 2003.

[22] D. N. Spergel, R. Bean, O. Doré et al., “Three-year Wilkinson Microwave Anisotropy Probe (WMAP) observations: implications for cosmology," The Astrophysical Journal, vol. 170, pp. 377-408, 2007.

[23] A. G. Reiss, L. G. Strolger, J. Tonry et al., “Type Ia Supernova Discoveries at $\mathrm{z} i 1$ from the Hubble Space Telescope: evidence for past deceleration and donstraints on dark energy evolution," The Astrophysical Journal, vol. 607, pp. 665-687, 2004.

[24] G. B. Zhao, R. G. Crittenden, L. Pogosian, and X. Zhang, "Examining the evidence for dynamical dark energy," Physical Review Letters, vol. 109, Article ID 171301, 5 pages, 2012.

[25] J. Q. Xia, Y. F. Cai, T. T. Qiu, G. B. Zhao, and X. Zhang, "Constraints on the sound speed of dynamical dark energy," International Journal of Modern Physics D, vol. 17, p. 1229, 2008.

[26] B. Feng, X. Wang, and X. Zhang, "Dark energy constraints from the cosmic age and supernova," Physics Letters B, vol. 607, pp. 35-41, 2005.

[27] Y.-F. Cai, H. Li, Y.-S. Piao, and X. Zhang, "Cosmic duality in quintom Universe," Physics Letters B, vol. 646, no. 4, pp. 141-144, 2007.
[28] Y. F. Cai and J. Wang, "Dark energy model with spinor matter and its quintom scenario," Classical and Quantum Gravity, vol. 25, no. 16, Article ID 165014, 2008.

[29] Y. F. Cai, E. N. Saridakis, M. R. Setare, and G. Q. Xia, "Quintom cosmology: theoretical implications and observations," Physics Reports, vol. 493, pp. 1-60, 2010.

[30] P. H. Frampton, K. J. Ludwickl, and R. J. Scherrer, "The little rip," Physical Review D, vol. 84, Article ID 063003, 5 pages, 2011.

[31] P. H. Frampton, K. J. Ludwick, and R. J. Scherrer, "Pseudo-rip: cosmological models intermediate between the cosmological constant and the little rip," Physical Review D, vol. 85, Article ID 083001, 5 pages, 2012.

[32] Y. S. Myung, "Quintessence with a localized scalar field on the brane," Modern Physics Letters A, vol. 16, no. 30, pp. 1963-1972, 2001.

[33] S. Carroll, An Introduction to General Relativity: Spacetime and Geometry, Addison Wesley, 2004.

[34] V. Faraoni, "Inflation and quintessence with nonminimal coupling," Physical Review D, vol. 62, no. 2, Article ID 023504, 15 pages, 2000.

[35] S. Nesseris and L. Perivolaropoulos, "Crossing the phantom divide: theoretical implications and observational status," Journal of Cosmology and Astroparticle Physics, vol. 2007, no. 01, article 018, 2007.

[36] A. Vikman, "Can dark energy evolve to the phantom?" Physical Review D, vol. 71, Article ID 023515, 14 pages, 2005.

[37] A. A. Starobinsky, "Future and origin of our Universe: modern view," Gravitation and Cosmology, vol. 6, no. 3, pp. 157-163, 2000.

[38] E. Elizalde, S. Nojiri, and S. D. Odintsov, "Late-time cosmology in a (phantom) scalar-tensor theory: dark energy and the cosmic speed-up," Physical Review D, vol. 70, Article ID 043539, 20 pages, 2004.

[39] K. Bamba, S. Nojiri, and S. D. Odintsov, "The future of the universe in modified gravitational theories: approaching a finitetime future singularity," Journal of Cosmology and Astroparticle Physics, vol. 2008, no. 10, article 045, 2008.

[40] S. Nojiri, S. D. Odintsov, and S. Tsujikawa, "Properties of singularities in (phantom) dark energy universe," Physical Review D, vol. 71, Article ID 063004, 17 pages, 2005.

[41] S. D. Sadatian, "Cosmological aspects of a vector field model," International Journal of Modern Physics D, vol. 21, Article ID 1250063, 18 pages, 2012.

[42] Arianto, F. P. Zen, Triyanta, and B. E. Gunara, "Attractor solutions in Lorentz violating scalar-vector-tensor theory," Physical Review D, vol. 77, no. 12, Article ID 123517, 13 pages, 2008.

[43] F. P. Zen, Arianto, B. E. Gunara, Triyanta, and A. Purwanto, "Cosmological evolution of interacting dark energy in Lorentz violation,” The European Physical Journal C, vol. 63, pp. 477-490, 2009.

[44] Arianto, F. P. Zen, and B. E. Gunara, "Modified gravitational equations on braneworld with Lorentz invariant violation," General Relativity and Gravitation, vol. 42, no. 4, pp. 909-927, 2010.

[45] V. A. Kostelecky and S. Samuel, "Spontaneous breaking of Lorentz symmetry in string theory," Physical Review D, vol. 39, pp. 683-685, 1986.

[46] S. D. Sadatian and K. Nozari, "Crossing of the phantom divided barrier with Lorentz invariance violating fields," Europhysics Letters, vol. 82, no. 4, Article ID 49001, 2008. 
[47] B. Li, D. F. Mota, and J. D. Barrow, "Detecting a Lorentzviolating field in cosmology," Physical Review D, vol. 77, no. 2, Article ID 024032, 13 pages, 2008.

[48] M. Libanov, V. Rubakov, E. Papantonopoulos, M. Sami, and S. Tsujikawa, "Ultraviolet stable, Lorentz-violating dark energy with transient phantom era," Journal of Cosmology and Astroparticle Physics, vol. 2007, no. 08, article 010, 2007.

[49] O. Bertolami, R. Lehnert, R. Potting, and A. Ribeiro, "Cosmological acceleration, varying couplings, and Lorentz breaking," Physical Review D, vol. 69, Article ID 083513, 11 pages, 2004.

[50] H. Wei and R.-G. Cai, "A note on crossing the phantom divide in hybrid dark energy model," Physics Letters B, vol. 634, pp. 9-13, 2006.

[51] R. Amanullah, C. Lidman, D. Rubin et al. et al., "Spectra and hubble space telescope light curves of six type Ia supernovae at $0.511<z<1.12$ and the union2 compilation"," The Astrophysical Journal, vol. 716, no. 1, p. 712, 2010.

[52] http://supernova.lbl.gov/.

[53] S. Nesseris and L. Perivolaropoulos, "Comparison of the legacy and gold type Ia supernovae dataset constraints on dark energy models," Physical Review D, vol. 72, Article ID 123519, 8 pages, 2005.

[54] K. Bamba, S. Capozziello, S. Nojiri, and S. D. Odintsov, "Dark energy cosmology: the equivalent description via different theoretical models and cosmography tests," Astrophysics and Space Science, vol. 342, no. 1, pp. 155-228, 2012. 

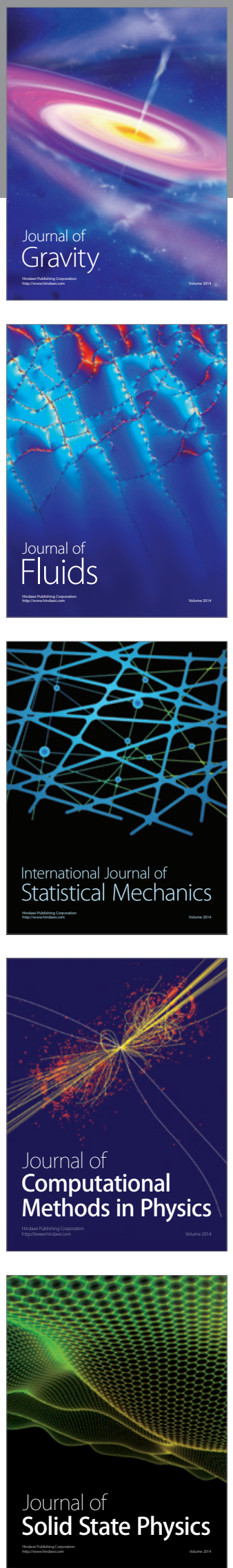

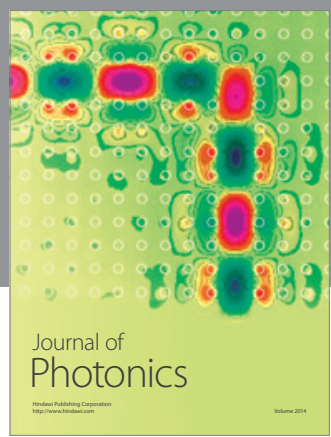

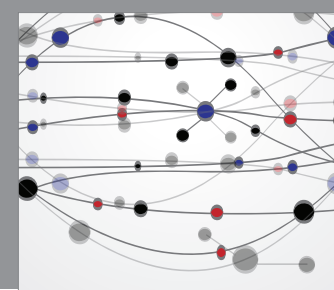

The Scientific World Journal

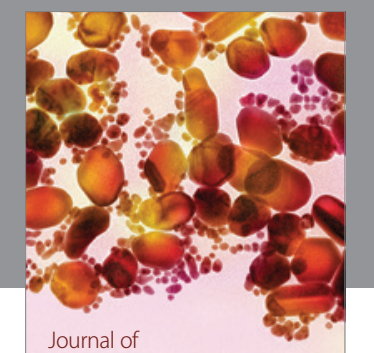

Soft Matter
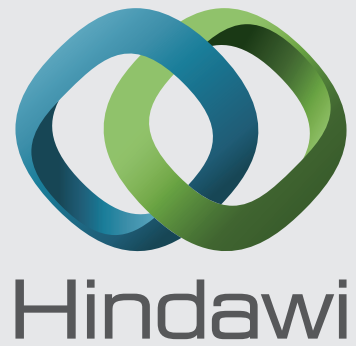

Submit your manuscripts at

http://www.hindawi.com
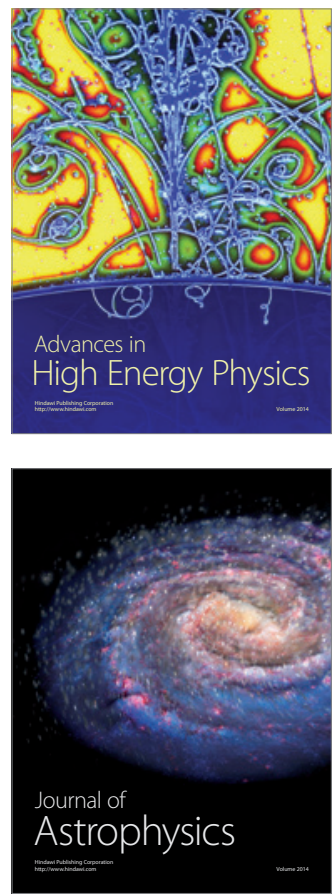
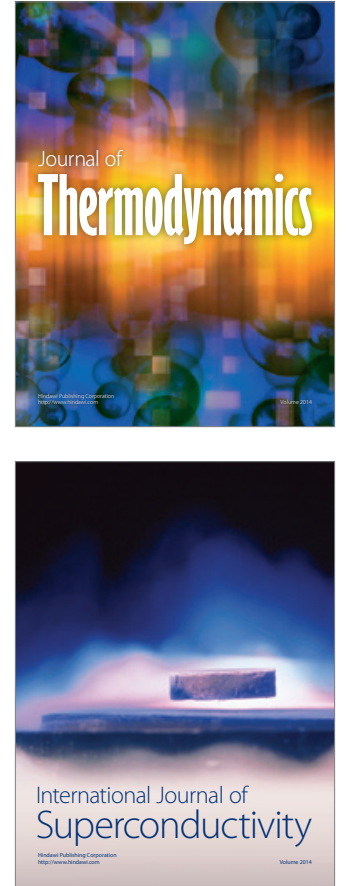
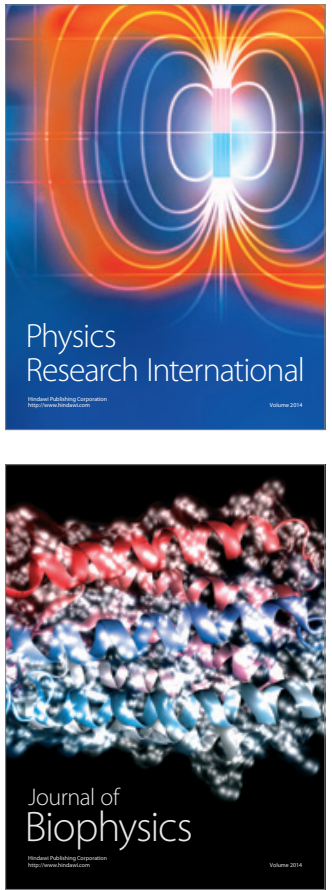
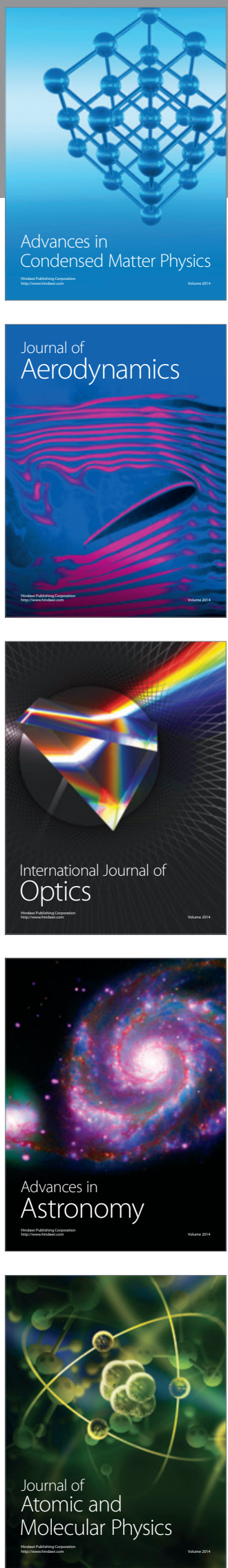